\title{
An experimental investigation of trailing-edge noise reduction due to elasticity
}

\author{
Maurício M. Nilton *, Yasir A. Malik ${ }^{\dagger}$, André V. G. Cavalieri ${ }^{\ddagger}$, \\ Leandro D. Santana § Maurício V. Donadon, \\ William R. Wolf" and Cristiano Pimenta**
}

August 24, 2018

\begin{abstract}
The proximity of the source and an edge can make the acoustic scattering by wings a significant source of aerodynamic sound. Theoretical results have shown that elastic edges lead to reductions of acoustic scattering; however, experimental confirmation of theoretical trends is difficult, since surface vibrations modify both the source structure and the scattering properties. A simplified, controlled setting for measurements of acoustic scattering, allowing the evaluation of fluid-structure interactions, would thus be desirable to study how elastic edges modify the radiated sound. We present an experimental procedure to isolate the scattered field using a loudspeaker in the vicinity of flat plates. The methodology is applied to three different plates, made of steel, aluminum and carbon fiber, as a demonstration. The responses of these elastic plates are studied for a sound source of dipole type near the trailing edge. The method is based on the experimental determination of frequency response functions between source and radiated sound for experiments with and without the plate; subtraction of results, accounting for amplitude and phase, isolates the scattered field. Experimental results treated with the developed procedure were compared with predictions made by numerical simulations performed with a Boundary Element Method (BEM), coupling the acoustic problem with the plate vibration. The comparison between experimental and numerical results revealed that a twodimensional model can predict satisfactorily the reductions in scattered field by elastic plates observed in the experiment. The present methods can be used to support the choice between different materials for edges focusing on their respective acoustic benefit.
\end{abstract}

\section{Introduction}

The generation of noise by solid bodies immersed in a flow was first investigated by Curle 1]. Using a dimensional analysis, it was shown that, in a regime whose Mach number is sufficiently low, the sound produced due to the presence of the solid surface is dominant. While Curle's analysis considers compact surfaces, the problem of acoustic scattering that occurs when an eddy is close to a trailing edge requires an approach that considers an airfoil acting as a non-compact source. Such trailing edge noise that occurs on a semi-infinite plate was studied by Powell[2], where dipole type sources were positioned in the vicinity of the trailing edge, providing an estimate of the radiated sound power spectral density. Ffowcs-Williams and Hall[3] also analyzed the interaction of the trailing edge of the plate with the disturbed flow. The estimate of the acoustical intensity in the far field showed that the presence of thin edges considerably impacts the increase of the aerodynamic noise generated in low Mach numbers. Besides the works cited above, there are other references 4, 5, 6, 7, 8, 9, that are also devoted to the problem of trailing edge noise; most often, the problem is studied theoretically or numerically.

The interaction of a sound source with the trailing edge of a plate gives rise to a scattered acoustic field which is added to the incident field generated by the source. Whenever modifcations of a plate are introduced, such as changes in geometry or in stiffness, effects on both source and scattering may occur. The source may change, as transitional or turbulent boundary layers close to the edge may be influenced by the aforementioned modifications; in addition to this, changes in acoustic scattering are expected, such as reductions in radiated

*PhD Student, Instituto Tecnológico de Aeronáutica, São José dos Campos, Brazil.

$\dagger$ M.Sc. Student, Universiteit Twente, Enschede, Netherlands

¥Assistant Professor, Divisão de Engenharia Aeronáutica, Instituto Tecnológico de Aeronáutica, Member AIAA.

$\S$ Assistant Professor, Department of Engineering Fluid Dynamics, Universiteit Twente, Enschede, Netherlands

ฯ Assistant Professor, Divisão de Engenharia Aeronáutica, Instituto Tecnológico de Aeronáutica, Member AIAA.

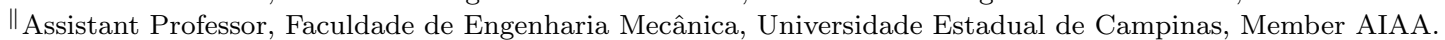

** PhD Student, Faculdade de Engenharia Mecânica, Universidade Estadual de Campinas 
sound due to elasticity and porosity effects [10, 8, 11. Obtaining the scattered field in a simplified setting, with a fixed, imposed source, may be interesting to characterize a particular wing or plate in relation to its scattering properties. However, the scattered field may be a small portion of the total field when a loudspeaker of monopole or dipole type is used as a source. Maybe due to these difficulties, to the best of our knowledge an experimental acquisition of the scattered field is lacking. In this paper, we present a method to isolate the scattered field for loudspeakers in the vicinity of elastic plates. Moreover, we use this method as an experimental study of the elasticity effect on acoustic scattering, with a comparison of measurements with numerical results obtained with a boundary element method. The present approach allows a controlled study of potential acoustic benefits of elastic edges.

The remainder of the paper is outlined as follows. An experiment, which was designed to demonstrate the operation of the data processing procedure is presented in section 2, and the signal processing is detailed in section 3 . A mathematical model for comparison and numerical procedures are briefly explained in section 4 Results and discussion are presented in section 5 and the paper is closed by the presentation of conclusions in section 6

\section{Experimental description}

In this section, we present an experiment that was designed to obtain edge scattering in a controlled setting, with a source given by a loudspeaker. For this, an incident field that corresponds to white noise will be used and we will analyze the acoustic field scattered by flat plates with different elasticity properties, i.e. made of different materials, namely aluminum, steel and carbon fiber.

\subsection{Apparatus}

The tests were conducted in the anechoic wind tunnel facility at the University of Twente. The wind tunnel has an semi-open test section measuring $0.9 \times 0.7 \mathrm{~m}^{2}$ in an anechoic chamber measuring $6 \times 6 \times 4 \mathrm{~m}^{3}$. All tests were conducted in the anechoic chamber without operation of the wind tunnel, and the ambient medium was thus at rest. The plates used in the tests were made from aluminum, carbon fiber and steel. They have a span $(w)$ of $0.7 \mathrm{~m}$ in $z$ direction, and a chord $(\ell)$ of $0.45 \mathrm{~m}$ in $x$ direction. Aluminium and steel plates have thickness equal to $1.0 \mathrm{~mm}$. The carbon fiber laminate has a thickness of $0.5 \mathrm{~mm}$. The plates were mounted in a structure inside the anechoic chamber. One of the edges was assigned as a "leading edge"; the plates were in a cantilever configuration, clamped at the leading edge with all other edges free, following a configuration studied in theoretical works $[8,11$. The loudspeaker was positioned in the vicinity of the trailing edge and microphones were used to measure the near and far field.

\subsection{Coordinate system and device positioning}

The origin of the coordinate system is at the leading edge. The loudspeaker was positioned at plate midspan, aligned with the trailing edge in the $x$ direction, and at a distance $d=8 \mathrm{~mm}$ in the $y$ direction. Microphone M1 was positioned $2 \mathrm{~mm}$ away from the loudspeaker in the direction $y$ to measure the near field pressure. The microphones M2 and M3 were positioned at a distance $D=2.4 \mathrm{~m}$ to measure the far field, M2 aligned with the trailing edge and M3 with a different angle, as shown in Figure 1.

\section{$3 \quad$ Signal processing}

We now present an approach to process the experimental data using the setup described in the previous section so as to isolate the scattered sound field. The method will be explained based on the cases studied here related to the evaluation of acoustic benefits due to plate elasticity, but the approach has broader application, and can be used in different situations, for example, to characterize the acoustic response of materials in design phases.

The first point to emphasize in this experiment is that besides measuring the acoustic pressure at selected points of observation with microphones, we would need a simultaneous measurement related to the source. It could be the input voltage to the loudspeaker or the near field pressure. Both have been attempted in this study, and were seen to lead to similar results. The near field pressure was measured with mircrophone M1, shown in Figure 1.

Hereafter we call one of the source measurements (voltage or near field pressure) as $X$ and the observer measurements (acoustic pressure from microphone M2 or M3) as $Y$. The frequency-response function (FRF) 


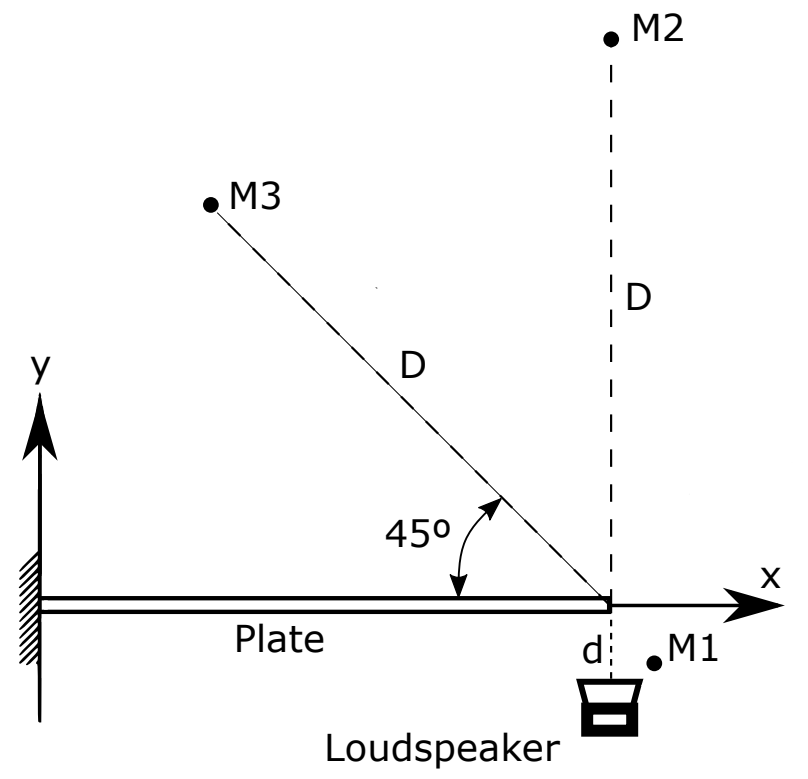

Figure 1: Sketch of the set up with clamped leading edge and free trailing edge (top view).

between source-observer, when the source is excited stochastically can be estimated as 12 .

$$
G(f)=\frac{S_{X Y}(f)}{S_{X X}(f)}
$$

where $S_{X Y}(f)$, the cross-spectral density between source $X$ and observer $Y$, is the Fourier transform of the crosscorrelation function, and $S_{X X}(f)$ is the power-spectral density of the source, given by $S_{X X}=|\hat{X}(f)|^{2}$, where $\hat{X}(f)$ is the Fourier transform of the signal and $f$ the frequency in Hz. Notice that $S_{X Y}$ is a complex-valued quantity, with amplitude and phase, whereas $S_{X X}$ is real and positive. $G(f)$ gives the expected amplitude and phase at the observer if the source excitation at frequency $f$ has amplitude equal to 1 .

The key to isolate the scattered field is to run an experiment without the plate to obtain $G_{i}(f)$, i.e. the FRF for the incident field; the setup to do this is shown in Figure 2(a). Then another experiment with the plate (Figure 2(b)) is performed to obtain $G_{t}(f)$, the FRF for the total field. The FRF of the scattered field, $G_{s}(f)$, is simply

$$
G_{s}(f)=G_{t}(f)-G_{i}(f),
$$

with subtraction of complex quantities, accounting for amplitudes and phases.

Following the procedure above, $G_{s}(f)$ can be obtained for different plates. These transfer functions should be different for the cases investigated, according to theory [5, 8. However, for a dipole source close to the edge, the total transfer functions $G_{t}(f)$ for different plates are dominated by the incident field and should be nearly identical. A meaningful quantity to compare to theory and highlight the differences between these cases would be choose a baseline case, and then compare,

$$
\left|G_{s, \text { sample }}\right|-\left|G_{s, \text { baseline }}\right|,
$$

which would show the acoustic benefit in the scattered sound as a baseline plate is replaced by other plate. In this study, we have chosen the steel plate as the baseline case, as it can be considered to be rigid if compared to the other plates.

In order to ensure that there are no background noise issues, it is necessary to be within the limits of linear acoustics and vibration and to avoid significant spurious noise in the measurements. This occurs when the coherence between $X$ and $Y$ is close to unity, which highlights the linearity of the FRF.

\section{Numerical Simulations}

As previously mentioned, the experiment performed in this study consists of flat plates being excited by a sound source in the vicinity of the trailing edge. It was designed in such a way that it is possible to compare its results 


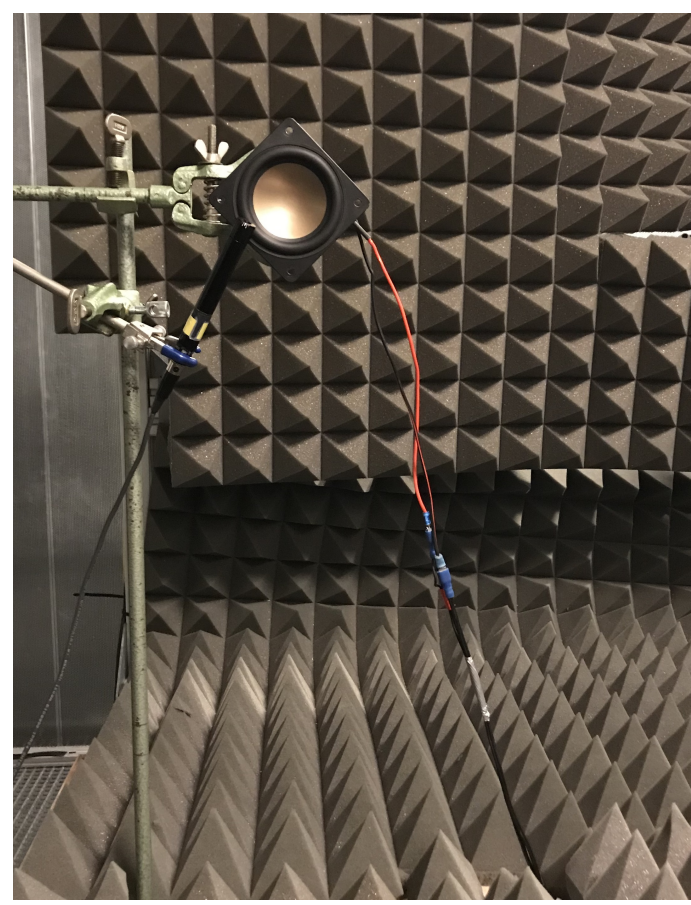

(a) Assembly without the plate to acquire the incident field generated by the loudspeaker

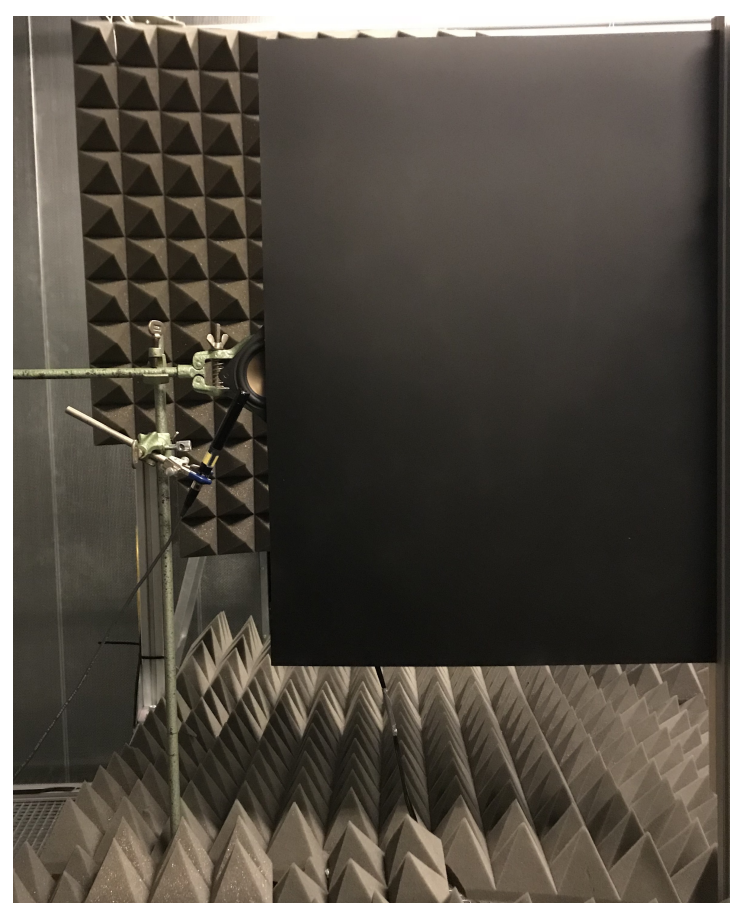

(b) Assembly with the plate to acquire the acoustic total field

Figure 2: Experimental set up mounted on the anechoic chamber

with those that are obtained numerically by a method that calculates the acoustic field scattered by finite elastic plates, described in Cavalieri et al. 8 and Nilton et al. 11. It is beyond the scope of this document to explain the complete method, but the essential aspects for understanding this study will be addressed in this section.

\subsection{Basic equations}

The problem at hand is a flat elastic plate, which is discretized into a finite number of elements, with a sound source $S$ positioned in the vicinity of the trailing edge. The sound source is modeled as a dipole to represent an unboxed loudspeaker, Figure 3(a) shows an incident field generated by a dipole source close to the plate. The model used is two-dimensional, so the plate has an infinite span in the direction $z$ and finite chord in the direction $x$. The plate boundary conditions corresponding to the experiment are: clamped leading edge and free trailing edge, as represented in Figure $3(\mathrm{~b})$.

To obtain the scattered pressure field, we solve the inhomogeneous non-dimensional Helmholtz equation,

$$
\nabla^{2} p+k_{0}^{2} p=-S
$$

where $k_{0}$ is the non-dimensional acoustic wavenumber, given as $\tilde{\omega} \ell / \tilde{c}_{0}$ for angular frequency $\tilde{\omega}$, characteristic length $\ell$ and speed of sound $\tilde{c}_{0}$. The overhead tildes indicate dimensional terms, and an $\exp (-i \tilde{\omega} \tilde{t})$ time dependence is implicitly assumed. The pressure $p$ is given as $\tilde{p} /\left(\tilde{\rho}_{f} \tilde{c}_{0}^{2}\right)$, where $\tilde{\rho}_{f}$ is the fluid density, and $S$ is the acoustic source function. Equation 4 is subject to boundary conditions matching velocities of the fluid and vibrating plate at the fluid-solid interface,

$$
\nabla^{4} \eta-\frac{k_{0}^{4}}{\Omega^{4}} \eta=\epsilon \frac{k_{0}^{3}}{\Omega^{6}} \Delta p
$$

where $\eta$ is the plate displacement, $\Omega$ is the vacuum bending wave Mach number and $\epsilon$ is the intrinsic fluid loading parameter. This is derived from the equation for a harmonic load $\Delta p$ applied to a thin elastic plate; details on the derivation can be found in the references [13, 11. Typical values of $\epsilon$ for steel and aluminium in air can be found in Howe [14. For the carbon fiber plate this value can be calculated as

$$
\epsilon=\frac{\tilde{\rho_{f}}}{\tilde{\rho_{s}}}\left(\frac{E}{12 \tilde{\rho_{s}} \tilde{c}_{0}^{2}\left(1-\nu^{2}\right)}\right)^{1 / 2},
$$




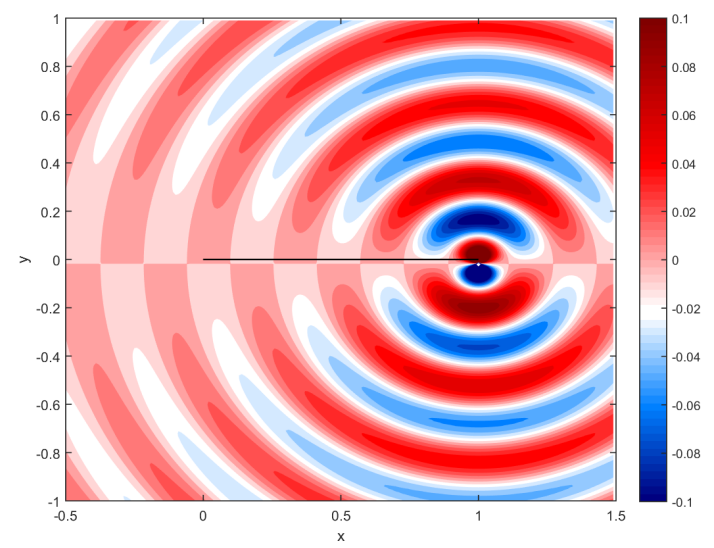

(a) Incident field generated by a dipole, acoustic wavenumber $k_{0}=20$.

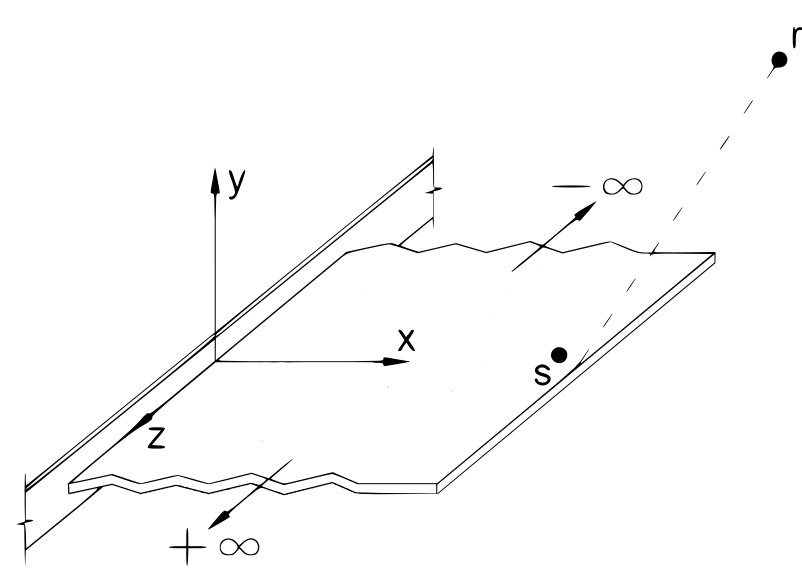

(b) Schematic of a rectangular elastic plate with finite chord and infinite span, where one edge is clamped along the $z$ axis and the other edge is free, subject to acoustic radiation from source $S$

Figure 3: Conditions used for numerical simulation.

where $E$ is the Young's modulus, $\tilde{\rho_{s}}$ is the mass density of the plate material and $\nu$ is the Poisson's ratio. Another important parameter in problems involving fluid-structure interaction is the relation $\omega_{c} h / c_{0}$, a non-dimensional coincidence frequency, given by,

$$
\frac{\omega_{c} h}{c_{0}}=\frac{\tilde{c}_{0}}{\tilde{c}_{1}}\left(\frac{12(1-\sigma)^{2}}{1-2 \sigma}\right)^{1 / 2} \equiv \frac{\tilde{\rho_{f}}}{\epsilon \tilde{\rho_{s}}} .
$$

We need another boundary condition to close the problem, and it is given by the linearised Euler equation, that relates the pressure and its normal derivative on the plate surface,

$$
k_{0}^{2} \eta=\left.\frac{\partial p}{\partial y}\right|_{y=0} .
$$

The acoustic problem is represented by Equation 4 subject to boundary conditions 5 and 8 It is necessary to provide two boundary conditions for the vibration problem 5 at each end of the plate to close the problem. For the cantilever plate configuration of Figure 3(b) these conditions are

$$
\eta(0)=\frac{\partial \eta(0)}{\partial x}=\frac{\partial^{2} \eta(1)}{\partial x^{2}}=\frac{\partial^{3} \eta(1)}{\partial x^{3}}=0 .
$$

This formulation is directly applicable to isotropic materials. The composite materials are characterized by their anisotropy, so to analyze them some modifications in the mathematical model are required; this specific treatment can be found in Cavalieri et al [15].

\subsection{Solution of problem using a structural modal basis}

The fluid-structure interaction problem is solved by rewriting equation 5 and substituting $\nabla^{4}$ for an operator $\mathcal{L}$. Now let us consider the auxiliary eigenvalue problem

$$
\mathcal{L}(\eta)=\beta^{4} \eta,
$$

subject to the same boundary conditions of equation 9 Solutions of this eigenvalue problem lead to a complete orthonormal basis $\phi_{i}$ for functions satisfying the boundary conditions of the problem, such that

$$
\mathcal{L}\left(\phi_{i}\right)=\beta_{i}^{4} \phi_{i}, \quad \text { where } \quad\left\langle\phi_{i}, \phi_{j}\right\rangle=\delta_{i j} .
$$

$\phi_{i}$ is called the modal basis; these modes are used as an auxiliary basis to solve the fluid-loaded plate problem. The eigenvalues of this problem are real and positive, and $\beta_{i}$ is identified as the bending wavenumber of a vibration mode $\phi_{i}$ of the plate.

Since the modal basis is a complete orthonormal set for functions satisfying the boundary conditions of the problem, we can use it to write the plate displacement $\eta$ and from equation 8 obtain the following equation, 
that relates the pressure difference between the two sides of the plate $\Delta p$ with the transverse pressure gradient evaluated at the plate surface $\partial p /\left.\partial y\right|_{y=0}$,

$$
\left.\frac{\partial p}{\partial y}\right|_{y=0}=\frac{\epsilon k_{0}^{5}}{\Omega^{6}} \frac{\left\langle\Delta p, \phi_{j}\right\rangle}{\beta^{4}-\frac{k_{0}^{4}}{\Omega^{4}}} \phi_{j}
$$

\subsection{Numerical methods}

The eigenvalue problem 11 is solved using a pseudo-spectral method 16 . The problem of acoustic scattering is solved by a boundary element method. A fundamental solution for the Helmholtz equation is the free space Green function, $\mathrm{G}(\mathbf{x}, \mathbf{y})$, written for a two-dimensional formulation as

$$
G(\mathbf{x}, \mathbf{y})=\frac{i}{4} H_{0}^{(1)}\left(k_{0}|\mathbf{x}-\mathbf{y}|\right)
$$

Here, $H_{0}^{(1)}$ stands for the Hankel function of the first kind and order zero. Using Green's second identity, one can write the following boundary integral equation

$$
T(\mathbf{x}) p(\mathbf{x})=\int_{\Gamma}\left[\frac{\partial p(\mathbf{y})}{\partial n_{y}} G(\mathbf{x}, \mathbf{y})-\frac{\partial G(\mathbf{x}, \mathbf{y})}{\partial n_{y}} p(\mathbf{y})\right] d \Gamma-\frac{\partial G\left(\mathbf{x}, \mathbf{z}_{i}\right)}{\partial \mathbf{z}_{i_{n}}} S\left(\mathbf{z}_{i}\right)
$$

where $T(\mathbf{x})=1 / 2$ when $\mathbf{x}$ is on a smooth boundary surface $\Gamma$, and $T(\mathbf{x})=1$ when $\mathbf{x}$ is a field point anywhere in the fluid region. The derivatives with respect to the inward normal direction of the boundary surface are represented by $\partial / \partial n$ and $n$ is an inward unit normal. The $i^{\text {th }}$ source location is $z_{i}$ and the incident dipolar field can be computed as the first derivative of the Green's function.

The scattering surface, $\Gamma$, is discretized into a finite number of elements with polynomial reconstructions for the unknowns in each element. Then, equation 14 is solved for each of these elements through the solution of a linear system of equations, written in compact form as

$$
[H]\{p\}-[G]\{\partial p / \partial n\}=\{S\}
$$

whose solution is possible since $\{p\}$ and $\{\partial p / \partial n\}$ are related by equation 12

\section{$5 \quad$ Results}

\section{$5.1 \quad$ Numerical results}

In this section we will present results found using the numerical method described above. For all configurations, a dipole source was positioned at the vicinity of the trailing edge. The free edge is located at $(x, y)=(1,0)$, and the dipole is placed at $(x, y)=(1,-0.0178)$. We calculate the pressure for observers in the acoustic field located 5.33 chords from the plate trailing edge, which corresponds to the positioning radius of the microphones in the experiment. In order to solve the problem at hand we need the parameters related to the fluid-structure interaction, $\epsilon$ and $\omega_{c} h / c_{0}$. For the cases studied here, the plates are immersed in air and the values of these parameters for aluminum and steel are available in the reference 14. For the carbon fiber laminate they can be calculated using equations 6 and 7, and the mechanical properties of the biaxial lamina, $E_{1}=51.83 \mathrm{GPa}$, $E_{2}=51.83 \mathrm{GPa}, G_{12}=2.82 \mathrm{GPa}, \nu_{12}=0.06$ and the laminate density, which is $1336 \mathrm{~kg} / \mathrm{m} 3$. The resulting parameters for three plates are shown in the table 1. The carbon fiber laminate is more flexible than the aluminum plate, which in turn is more flexible than the steel plate. This reflects on the fluid loading parameter $\epsilon$, which is expected to be higher as elasticity is increased.

Table 1: Bending stiffness and fluid-structure parameters of plates in air

\begin{tabular}{ccc} 
Plate & $\omega_{c} h / c_{0}$ & $\epsilon_{0}$ \\
\hline Steel & 0.22 & 0.00073 \\
Aluminum & 0.22 & 0.0021 \\
Carbon fiber laminate & 0.1904 & 0.0049
\end{tabular}

For each of the three plates, we performed a sweep in $k_{0}$. To evaluate the effect of flexibility in acoustic scattering, Figure 4 show the scattered sound power level (PWL) of the three cases. In this figure and hereafter, CFP stands for carbon fiber composite plate. The first point to be observed is the acoustic benefit, with noise 


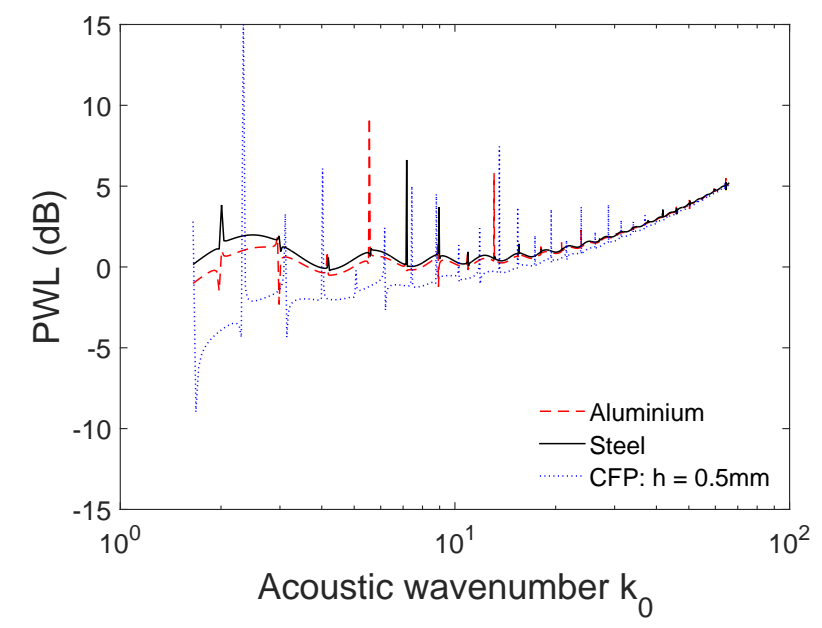

Figure 4: Sound power level radiated by elastic plates

reduction as we move from steel plate to aluminium plate and then to carbon fiber laminate; as elasticity is increased, reductions of acoustic scattered are observed, in agreement with previous works 14, 8, 10.

For each value of $k_{0}$, there is an associated value of $\Omega$, given by,

$$
\Omega=\sqrt{\frac{k_{0}(h / \ell)}{\omega_{c} h / c_{0}}} .
$$

The variation of $\Omega$ for the three plates is shown in the Figure 5, which is basically a reproduction of Figure 4. but each chart contains only one plate and at the top one can see a scale of $\Omega$. Note that for the steel and aluminum plates, the ranges of $\Omega$ are identical since these plates have the same thickness and the parameter $\omega_{c} h / c_{0}$ is also the same. By the joint analysis of figures 4 and 5 , it can be observed that there are more significant reductions of PWL as $\Omega$ is decreased, which is consistent with results for semi-infinite plates 7 . However, it is important to note that $k_{0}>1$, since the opposite condition, $k_{0}<1$ leads to lower reduction of PWL, as demonstrated in reference [8]. Another feature that can be observed is the presence of sharp peaks, which correspond to resonances of the fluid-loaded plates. As $\Omega$ is reduced the acoustic excitation frequency crosses successive resonance conditions.

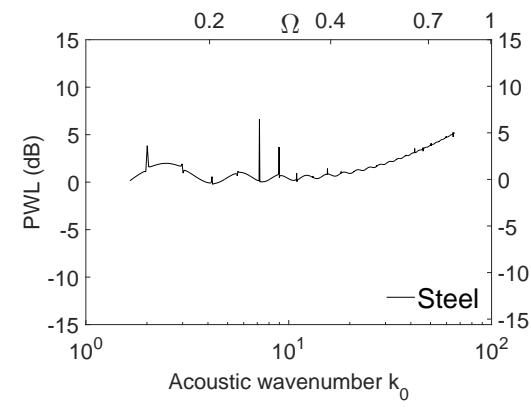

(a) Sound power level radiated by steel plate

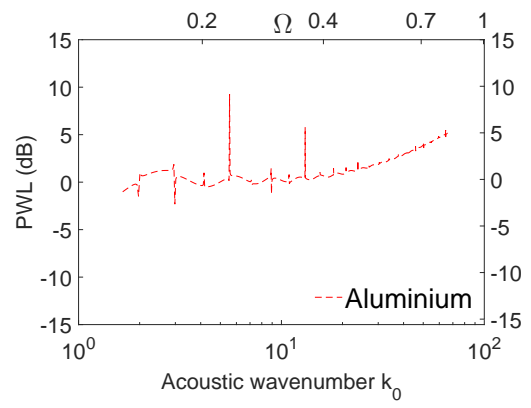

(b) Sound power level radiated by aluminium plate

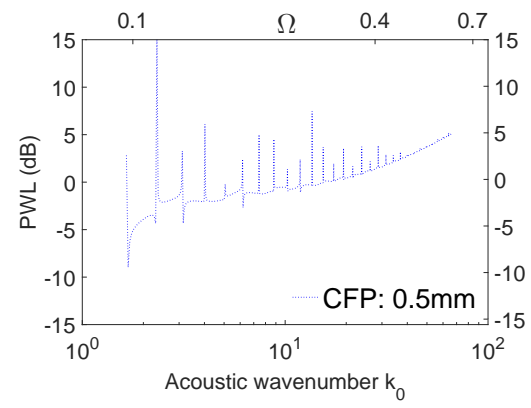

(c) Sound power level radiated by carbon fiber composite plate

Figure 5: Sound power level radiated by elastic plates, attention to the $\Omega$ scale in charts top.

Another way to present the results is to show the sound pressure level (SPL) in the observer positions corresponding to the microphones M2 (90 degrees to the x-axis) and M3 (135 degrees to the x-axis). We are interested in the acoustic benefit of one plate in relation to the others, so the Figure 6 shows the variation of sound pressure level $(\triangle S P L)$ in relation to the steel plate. Reductions of up to $5 \mathrm{~dB}$ are expected for the composite plate compared to the baseline case. 


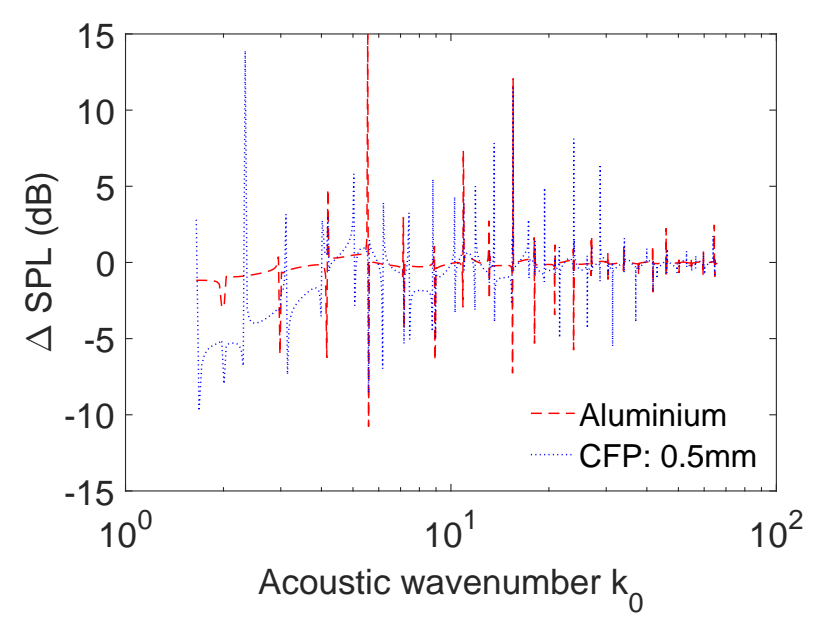

(a) At M2 microphone observer position $\left(90^{\circ}\right)$

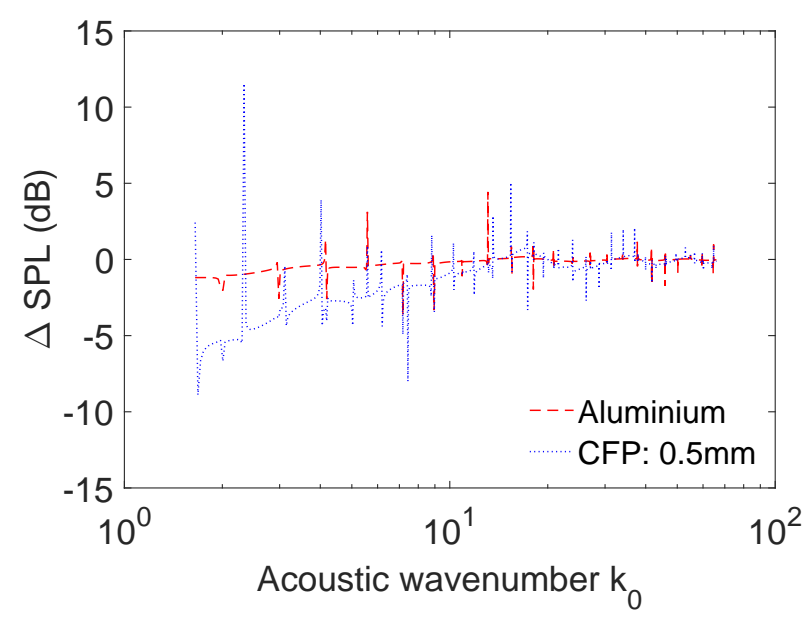

(b) At M3 microphone observer position $\left(135^{\circ}\right)$

Figure 6: Difference in scattered field amplitude with respect to the scattered field of the steel plate, calculated numerically.

\subsection{Experimental results}

As mentioned in section 3 , two signals were acquired to characterize the source: one measured by the microphone M1 and also the input voltage in the loudspeaker. The results that we present in this section were obtained using the input voltage signal to the loudspeaker, the results obtained with the microphone M1 are similar and will not be shown for the sake of brevity.

In a linear noise-free system, the frequency content of the output is the same as the frequency content of the input; only the magnitudes and phases of the frequency components are altered by the system. The coherence function for the input and output of a linear noise-free system is unity, indicating maximum or complete coherence. In this experiment, the coherence between the observer and the acoustic source was verified. Results for the microphone M2 are shown in the Figure 7 , and for M3 in the Figure 8.

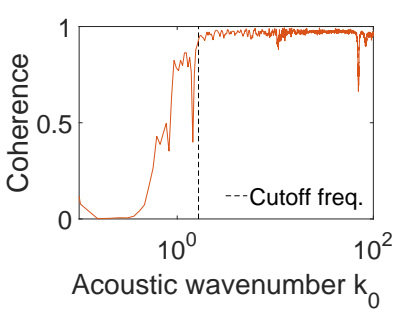

(a) Incident field

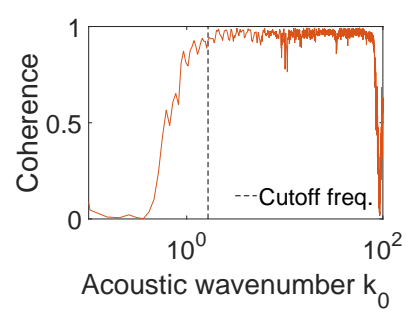

(b) Steel plate

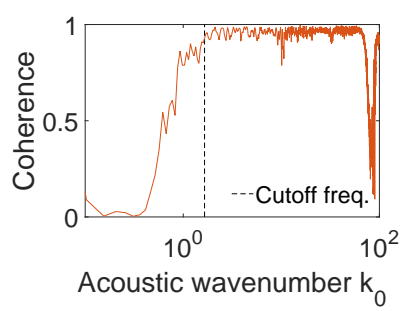

(c) Aluminium plate

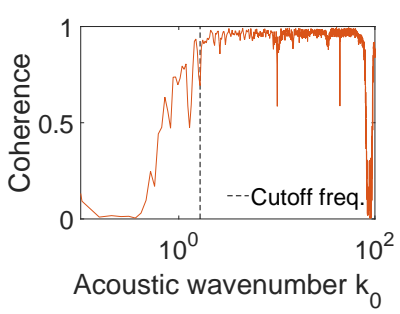

(d) Carbon fiber laminate

Figure 7: Coherence between source and microphone M2.

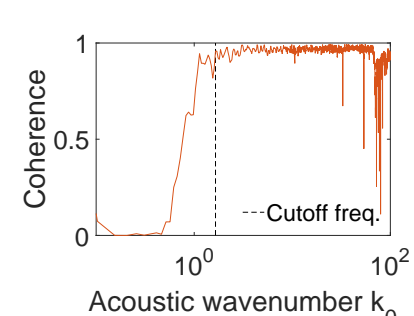

(a) Incident field

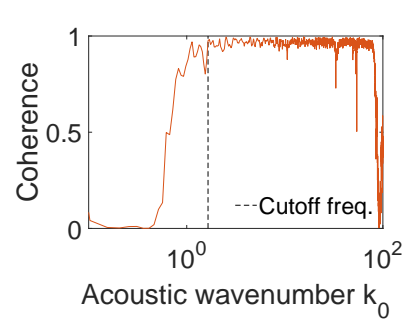

(b) Steel plate

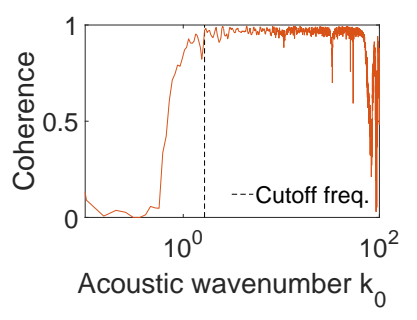

(c) Aluminium plate

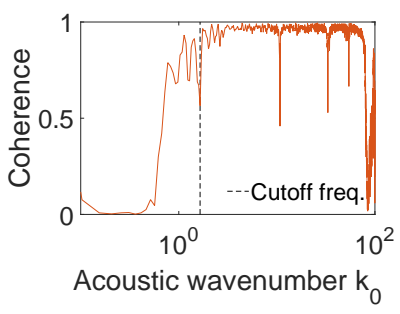

(d) Carbon fiber laminate

Figure 8: Coherence between source and microphone M3.

The analysis of figures 7 and 8 indicates that there is a range of values of $k_{0}$, starting at the anechoic chamber cutoff frequency $\left(200 \mathrm{~Hz}\right.$, corresponding to approximately $\left.k_{0}=1.65\right)$, where the coherence is close to one. To minimize the effect of background noise, we will only work with the acoustic wavenumber spectrum where the 
coherence is close to the unit value, that is, from $k_{0}=1.65$ to 66 , which corresponds to a frequency band from $200 \mathrm{~Hz}$ to $8000 \mathrm{~Hz}$.

The experiment was performed as described in section 2 and the data was processed following the procedure described in section 3. From this method it was possible to obtain the acoustic field scattered by the three plates in two observer positions, corresponding to the M2 and M3 microphones. This result is shown in Figure 9.

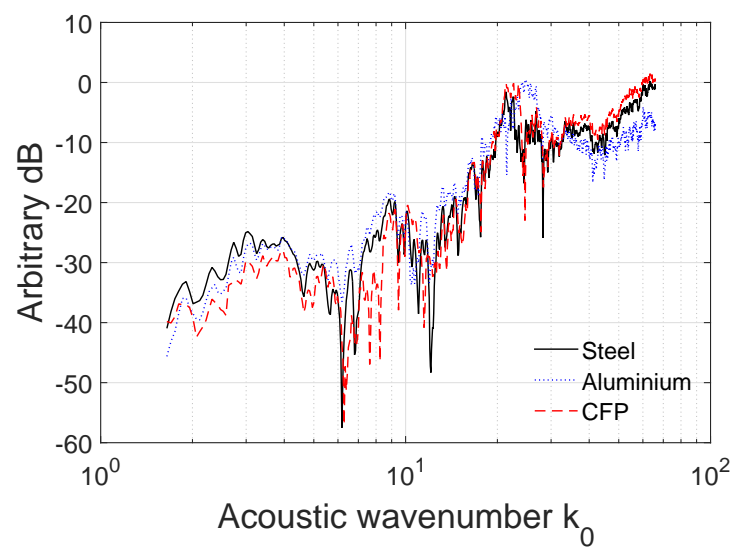

(a) Measured by microphone M2 $\left(90^{\circ}\right)$

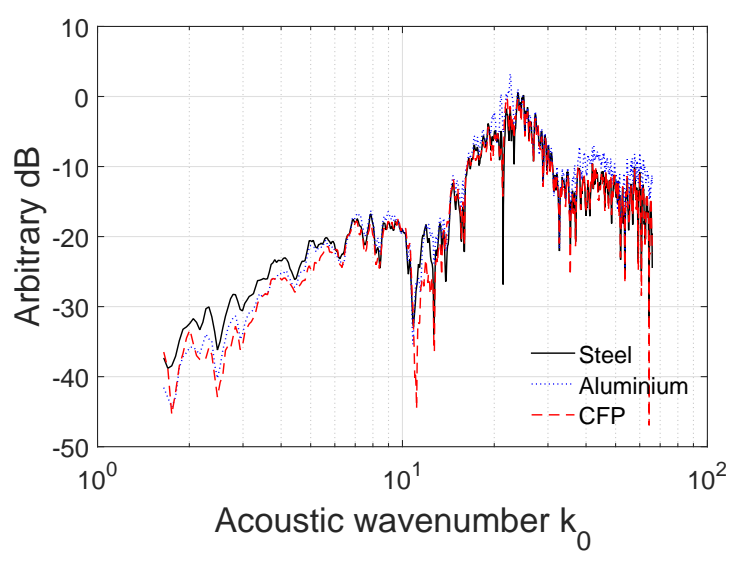

(b) Measured by microphone M3 $\left(135^{\circ}\right)$

Figure 9: Acoustic field scattered by the aluminum, carbon fiber and steel plates

To make these results comparable to those obtained by numerical calculations, we will present them in terms of the difference in sound pressure level $(\triangle S P L)$ with respect to the steel plate, which was chosen as the reference case. To obtain $\triangle S P L$ the following equation is used,

$$
\Delta S P L=20 \log \left(\left|\left(G_{s, \text { sample }}\right)\right|\right)-20 \log \left(\left|\left(G_{s, \text { baseline }}\right)\right|\right) .
$$

Results are shown in Figure 10, where it is possible to observe, in general, that when we replace the steel plate by the carbon fiber laminate we have greater reductions in the scattered acoustic field than when this replacement is made by the aluminum plate. For the composite plate, reductions of up to $5 \mathrm{~dB}$ are seen for lower values of $k_{0}$, in agreement with what was seen in the numerical results. In the following section the numerical and experimental results will be compared.

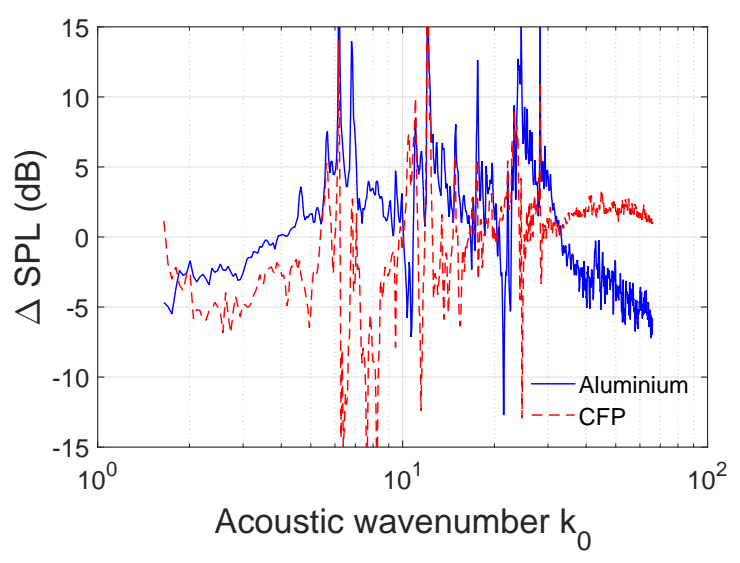

(a) Measured by microphone M2 $\left(90^{\circ}\right)$

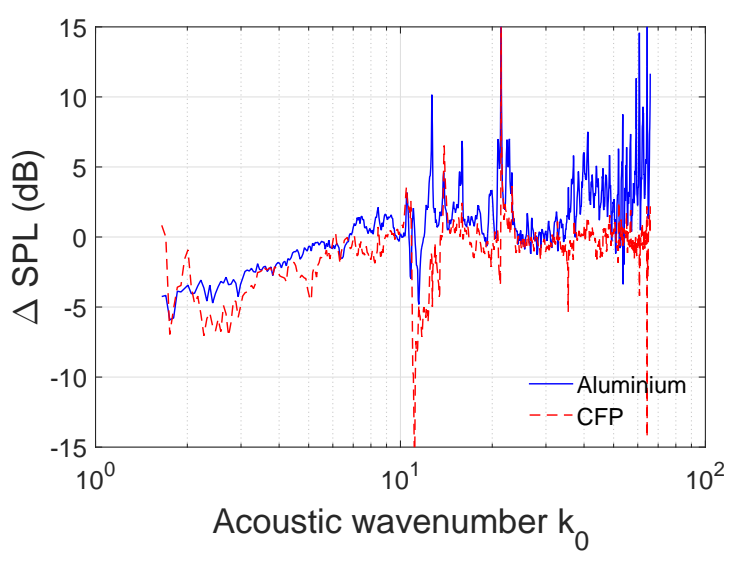

(b) Measured by microphone M3 $\left(135^{\circ}\right)$

Figure 10: Difference in scattered field amplitude with respect to the scattered field of the steel plate, measured experimentally. 


\subsection{Comparison of results}

In this section we present a comparison of numerical and experimental results. Figures 11 and 12 show the variations of sound pressure level in the observers positions corresponding to the microphones M2 and M3, respectively. The graphs related to the carbon fiber laminate indicate that the model was able to predict the general trends that were observed in the experiment. For example, if we look at Figure 11(b) for $k_{0}$ approximately equal to 8.0, we see a drop in the experimental graph that was correctly predicted by the numerical calculation. Other similar trends can be observed. However, for the aluminum plate, we observed in the numerical results that the minor difference in scattered field compared to the steel plate is likely difficult to be captured in the experiment.

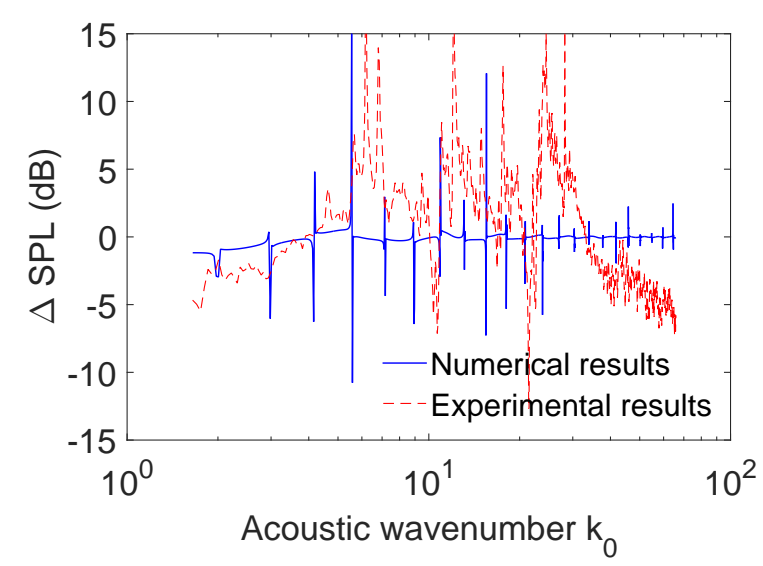

(a) Aluminium plate

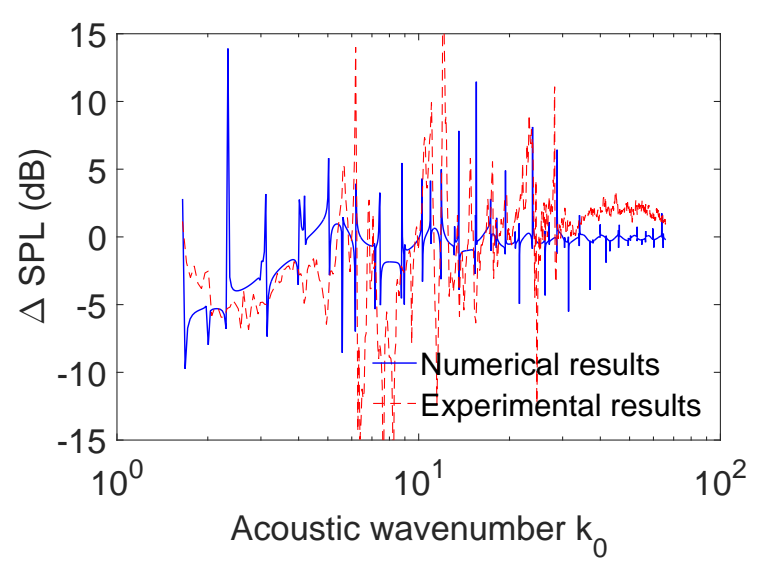

(b) Carbon fiber laminate

Figure 11: Difference in scattered field amplitude with respect to the scattered field of the steel plate, at microphone M2 observer position $\left(90^{\circ}\right)$.

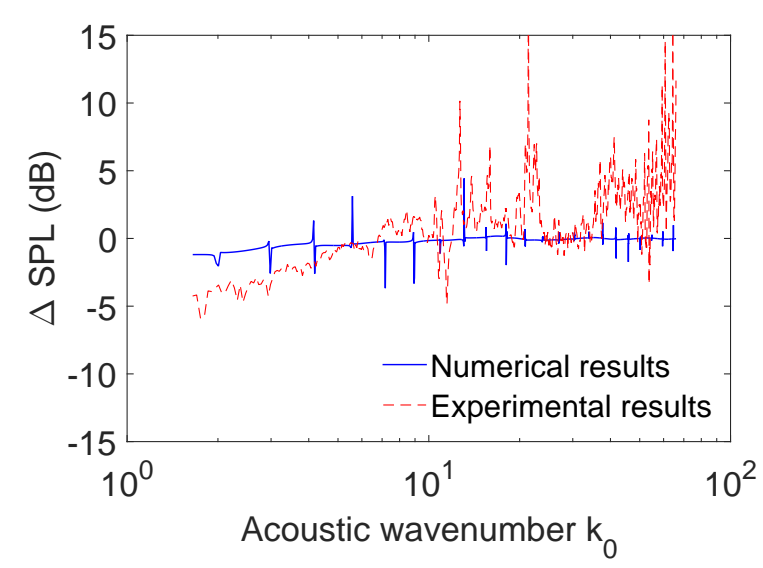

(a) Aluminium plate

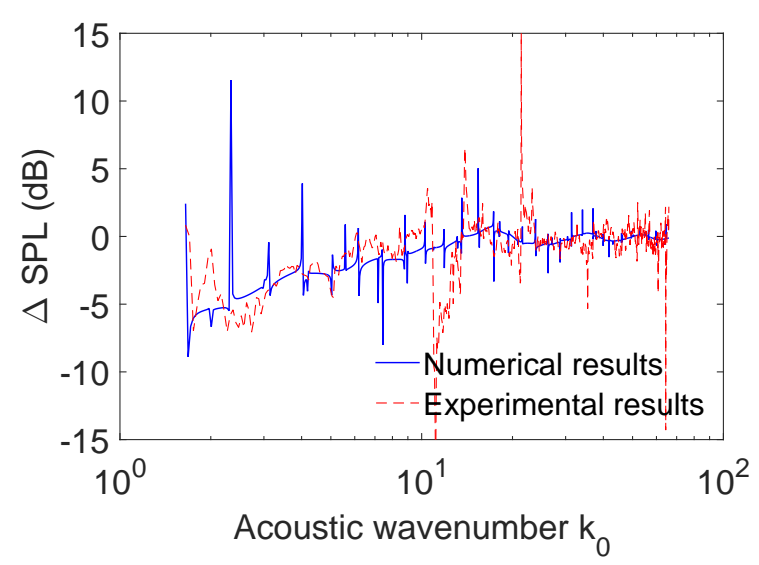

(b) Carbon fiber laminate

Figure 12: Difference in scattered field amplitude with respect to the scattered field of the steel plate, at microphone M3 observer position $\left(135^{\circ}\right)$.

\section{Conclusions}

We presented a procedure for signal processing focused on the experimental isolation of the scattered acoustic field for a setup with a loudspeaker in the vicinity of a plate edge. To prove the efficacy of the method, an experiment was designed, in which the scattered fields by flexible plates were analyzed. The overall behaviour observed here highlights that the effect of flexibility leads to reductions of the radiated sound. This is a proof of concept for the present approach; it could be extended to other applications focused on the analysis of the acoustics of fluid-structure interaction. 
Experimental results are similar when we consider the signal measured by the microphone in the near field or the voltage in the loudspeaker as the system input. Moreover, as a form of validation, we found that the results remain unchanged when we vary the interval in the time series, the number of discrete points in the Fourier transform, the overlap and also the way of estimating frequency response functions.

Numerical results for the problem were obtained by means of a formulation based on the boundary element method. The modal basis of the free-vibration problem is calculated numerically with a pseudo-spectral method, in a second step the acoustic problem is solved by a boundary element solver, which uses the previously obtained modal basis to relate $\partial p / \partial n$ on the plate surface to the pressure difference across the plate. The acoustic problem was modeled in two dimensions, and despite this limitation, when comparing numerical and experimental results it is observed that it is able to predict satisfactorily the trends observed in the experiment. This suggests that the main trends in trailing-edge noise may be reasonably predicted using a simpler, two-dimensional acoustic problem.

\section{Acknowledgments}

This work has been funded by the UTwentes International Strategic Partner Project Bandeirantes and the 4TU FSM center. The authors would like to thank S. Wanrooij and H. Stobbe for the technical support. Maurício M. Nilton was funded by a CAPES PhD scholarship. André V. G. Cavalieri was supported by a CNPq research scholarship.

\section{References}

[1] N. Curle, "The influence of solid boundaries upon aerodynamic sound," Proc. R. Soc. Lond. A, vol. 231, no. 1187, pp. 505-514, 1955.

[2] A. Powell, "On the aerodynamic noise of a rigid flat plate moving at zero incidence," The Journal of the Acoustical Society of America, vol. 31, no. 12, pp. 1649-1653, 1959.

[3] J. F. Williams and L. Hall, "Aerodynamic sound generation by turbulent flow in the vicinity of a scattering half plane," Journal of Fluid Mechanics, vol. 40, no. 4, pp. 657-670, 1970.

[4] R. K. Amiet, "Noise due to turbulent flow past a trailing edge," Journal of sound and vibration, vol. 47, no. 3, pp. 387-393, 1976.

[5] D. Crighton and F. Leppington, "Scattering of aerodynamic noise by a semi-infinite compliant plate," Journal of Fluid Mechanics, vol. 43, no. 4, pp. 721-736, 1970.

[6] D. Crighton, "Radiation from vortex filament motion near a half plane," Journal of Fluid Mechanics, vol. 51, no. 2, pp. 357-362, 1972.

[7] M. Howe, "Structural and acoustic noise produced by turbulent flow over an elastic trailing edge," in Proceedings of the Royal Society of London A: Mathematical, Physical and Engineering Sciences, vol. 442, pp. 533-554, The Royal Society, 1993.

[8] A. Cavalieri, W. Wolf, and J. Jaworski, "Numerical solution of acoustic scattering by finite perforated elastic plates," in Proc. R. Soc. A, vol. 472, p. 20150767, The Royal Society, 2016.

[9] W. R. Wolf and A. V. Cavalieri, "A fast numerical framework for acoustic scattering by $3 \mathrm{~d}$ poroelastic plates," in 21st AIAA/CEAS Aeroacoustics Conference, p. 3260, 2015.

[10] J. W. Jaworski and N. Peake, "Aerodynamic noise from a poroelastic edge with implications for the silent flight of owls," Journal of Fluid Mechanics, vol. 723, pp. 456-479, 2013.

[11] M. M. Nilton, A. V. Cavalieri, M. V. Donadon, and W. R. Wolf, "Effects of structural damping on acoustic scattering by flexible plates," in 23rd AIAA/CEAS Aeroacoustics Conference, p. 3204, 2017.

[12] J. S. Bendat and A. G. Piersol, Random data: analysis and measurement procedures, vol. 729. John Wiley \& Sons, 2011.

[13] A. V. Cavalieri, W. R. Wolf, and J. W. Jaworski, "Acoustic scattering by finite poroelastic plates," in 20th AIAA/CEAS Aeroacoustics Conf., Atlanta, GA, 2014. 
[14] M. S. Howe, Acoustics of fluid-structure interactions. Cambridge university press, 1998.

[15] A. V. Cavalieri, M. V. Donadon, and W. Wolf, "Acoustic scattering by finite composite plates," in 21st AIAA/CEAS Aeroacoustics Conference, p. 3262, 2015.

[16] L. N. Trefethen, Spectral methods in MATLAB, vol. 10. Siam, 2000. 